\title{
Recent QFT of Einstein's Gravity Simplified via Ultrahyperfunctions
}

\author{
A. Plastino ${ }^{1,2,3 *}$, M. C. Rocca ${ }^{1,2,4}$ \\ ${ }^{1}$ Departamento de Física, Universidad Nacional de La Plata, La Plata, Argentina \\ ${ }^{2}$ Consejo Nacional de Investigaciones Cientícas y Tecnológicas, La Plata, Argentina \\ 3 (IFLP-CCT-CONICET)-C. C., La Plata, Argentina \\ ${ }^{4}$ Departamento de Matemática, Universidad Nacional de La Plata, La Plata, Argentina \\ Email: *angeloplastino@gmail.com
}

How to cite this paper: Plastino, A. and Rocca, M.C. (2020) Recent QFT of Einstein's Gravity Simplified via Ultrahyperfunctions. Journal of Modern Physics, 11, 565-580.

https://doi.org/10.4236/jmp.2020.114037

Received: March 22, 2020

Accepted: April 11, 2020

Published: April 14, 2020

Copyright (C) 2020 by author(s) and Scientific Research Publishing Inc. This work is licensed under the Creative Commons Attribution International License (CC BY 4.0).

http://creativecommons.org/licenses/by/4.0/

\begin{abstract}
Previously published treatment is rather involved. Here we present a useful approximation to the concomitant derivation that yields a simpler way of handling things and still obtains results quite similar to those yielded by the exact treatment. Our approximation consists of giving the graviton field a simpler, but still quite good approximate form.
\end{abstract}

\section{Keywords}

Quantum Field Theory, Einstein Gravity, Non-Renormalizable Theories, Unitarity

\section{Introduction}

Previous attempts to quantize Einstein gravity (EG) have failed because of 1) they utilize a Rigged Hilber Space (RHS) with undefined metric, 2) non-unitarity troubles, and 3) non-renormalizablity problems. These problems were successfully bypassed in [1] [2], yielding a viable quantification procedure for Einstein's gravity. One used two kinds of tools. Some are of physical nature and were developed in [3] [4] [5]. Others are of a purely mathematical character, and concern the nature and properties of a generalization of Schwartz's distributions made in the 1960s. The generalized entities were called Ultrahyperfuntions (UHF). The existence and uniqueness of their convolution, whcih yields another UHF, were demonstrated in the 1990s [6]-[10]. Such convolutions are finite. Since quantum propagators can be seen to be UHFs, and their convolution is finite, all Feynman diagrams also turn out to be finite. There is no longer a need for renormalization. These important mathematical advanced were founded on the efforts 
of A. Grothendieck [11] and J. S. Silva (JSS) [12]. The concomitant mathematical apparatus was specifically devised so as to quantify non-renormalizable field theories. For a detailed discussion of it, see [10]. One ends up facing a theory similar to QED, endowed with unitarity at all finite orders of power expansions in the gravitation constant $G$ of the EG Lagrangian. This feat was previously attempted, but without success, by Gupta and by Feynman (in his celebrated Acta Physica Polonica paper [13]).

Our paper is structured as indicated below:

1) Section 2 deals with preliminary materials.

2) Section 3 treats the QFT Lagrangian for EG and introduces our approximation that consists of setting the graviton field $\phi^{\mu v}=\gamma^{\mu v} \phi$, where $\gamma^{\mu v}$ is a constant tensor and $\phi$ a scalar (graviton) field.

3) Section 4 quantizes the ensuing theory.

4) Section 5 evaluates the graviton's self-energy up to second order.

5) Section 6 introduces axions into our scenario and considers the axionsgravitons interaction.

6) Section 7 calculates the graviton's self-energy in the presence of axions.

7) Section 8 evaluates the axion's self-energy, up to second order.

8) Finally, some conclusions are drawn in Section 9.

\section{Preliminary Materials}

The most general quantification approach is based upon Schwinger-Feynman's variational principle [14] and can successfully deal even with high order supersymmetric (HOS) theories (see [15] [16]). It is important to emphasize, for QFT experts, that HOS theories cannot be quantized by appeal to the customary Dirac-brackets approach.

Consider now the action for a set of fields $\phi_{A}(x)$ written in the fashion

$$
\begin{aligned}
& \mathcal{S}\left[\sigma(x), \sigma_{0}, \phi_{A}(x)\right] \\
& =\int_{\sigma_{0}}^{\sigma(x)} L\left[\phi_{A}(\xi), \partial_{\mu} \phi_{A}(\xi), \xi\right] \mathrm{d} \xi,
\end{aligned}
$$

where $\sigma(x)$ if a space-like surface passing through the point $x . \sigma_{0}$ is that surface (at the remote past), for which all field variations vanish. The Schwinger-Feynman variational principle asserts then that:

"Any Hermitian infinitesimal variation $\delta \mathcal{S}$ of the action induces a canonical transformation of the vector space in which the quantum system is defined, and the generator of this transformation is this same operator $\delta \mathcal{S}$ ".

In such circumstances, this equality is seen to hold

$$
\delta \phi_{A}=i\left[\delta \mathcal{S}, \phi_{A}\right] .
$$

Accordingly, for a Poincare transformation one has 


$$
\delta \mathcal{S}=a^{\mu} \mathcal{P}_{\mu}+\frac{1}{2} a^{\mu v} \mathcal{M}_{\mu v},
$$

where $a^{\mu}$ and $a^{\mu \nu}$ are variational constants, the first for displacement and the second for rotation, $\mathcal{P}_{\mu}$ refers to linear momentum, and $\mathcal{M}_{\mu v}$ to complete angular momentum. For the field variation the last two quantities are represented by $\hat{P}_{\mu}$ and $\hat{M}_{\mu v}$, respectively. One has

$$
\delta \phi_{a}=a^{\mu} \hat{P}_{\mu} \phi_{A}+\frac{1}{2} a^{\mu v} \hat{M}_{\mu v} \phi_{A} .
$$

From (2.2) one can now ascertain that

$$
\partial_{\mu} \phi_{A}=i\left[\mathcal{P}_{\mu}, \phi_{A}\right] \text {. }
$$

More to the point,

$$
\partial_{0} \phi_{A}=i\left[\mathcal{P}_{0}, \phi_{A}\right]
$$

Equation (2.6) will be used below for quantizing EG.

\section{The Convolution of Two Lorentz Invariant Tempered Ultradistributions}

In [7] we have obtained a conceptually simple but rather lengthy expression for the convolution of two Lorentz invariant tempered ultradistributions. $\rho$ and $\Lambda$ are defined there. $\Theta$ is the Heaviside function and $\mathfrak{I}$ stands for imaginary part. We have then

$$
\begin{aligned}
& H_{\lambda}(\rho, \Lambda) \\
& \left.=\frac{1}{8 \pi^{2} \rho_{\Gamma_{1} \Gamma_{2}}} \int f_{1}\right) G\left(\rho_{2}\right) \rho_{1}^{\lambda} \rho_{2}^{\lambda}\left\{\Theta [ \Im ( \rho ) ] \left\{\left[\ln \left(-\rho_{1}+\Lambda\right)-\ln \left(-\rho_{1}-\Lambda\right)\right]\right.\right. \\
& \times\left[\ln \left(-\rho_{2}+\Lambda\right)-\ln \left(-\rho_{2}-\Lambda\right)\right] \sqrt{4\left(\rho_{1}+\Lambda\right)\left(\rho_{2}+\Lambda\right)-\left(\rho-\rho_{1}-\rho_{2}-2 \Lambda\right)^{2}} \\
& \times \ln \left[\frac{\sqrt{4\left(\rho_{1}+\Lambda\right)\left(\rho_{2}+\Lambda\right)-\left(\rho-\rho_{1}-\rho_{2}-2 \Lambda\right)^{2}}-i\left(\rho-\rho_{1}-\rho_{2}-2 \Lambda\right)}{2 \sqrt{\left(\rho_{1}+\Lambda\right)\left(\rho_{2}+\Lambda\right)}}\right] \\
& +\left[\ln \left(\rho_{1}+\Lambda\right)-\ln \left(\rho_{1}-\Lambda\right)\right]\left[\ln \left(\rho_{2}+\Lambda\right)-\ln \left(\rho_{2}-\Lambda\right)\right] \\
& \times \sqrt{4\left(\rho_{1}-\Lambda\right)\left(\rho_{2}-\Lambda\right)-\left(\rho-\rho_{1}-\rho_{2}+2 \Lambda\right)^{2}} \\
& \times \ln \left[\frac{\sqrt{4\left(\rho_{1}-\Lambda\right)\left(\rho_{2}-\Lambda\right)-\left(\rho-\rho_{1}-\rho_{2}+2 \Lambda\right)^{2}}-i\left(\rho-\rho_{1}-\rho_{2}+2 \Lambda\right)}{2 \sqrt{\left(\rho_{1}-\Lambda\right)\left(\rho_{2}-\Lambda\right)}}\right] \\
& +\left[\ln \left(\rho_{1}+\Lambda\right)-\ln \left(\rho_{1}-\Lambda\right)\right]\left[\ln \left(-\rho_{2}+\Lambda\right)-\ln \left(-\rho_{2}-\Lambda\right)\right] \\
& \times\left\{\frac{i \pi}{2}\left[\sqrt{4\left(\rho_{1}+\Lambda\right)\left(\rho_{2}-\Lambda\right)-\left(\rho-\rho_{1}-\rho_{2}\right)^{2}}-i\left(\rho-\rho_{1}-\rho_{2}\right)\right]\right. \\
& +\sqrt{4\left(\rho_{1}+\Lambda\right)\left(\rho_{2}-\Lambda\right)-\left(\rho-\rho_{1}-\rho_{2}\right)^{2}}
\end{aligned}
$$




$$
\begin{aligned}
& \left.\times \ln \left[\frac{\sqrt{4\left(\rho_{1}+\Lambda\right)\left(\rho_{2}-\Lambda\right)-\left(\rho-\rho_{1}-\rho_{2}\right)^{2}}-i\left(\rho-\rho_{1}-\rho_{2}\right)}{2 i \sqrt{-\left(\rho_{1}+\Lambda\right)\left(\rho_{2}-\Lambda\right)}}\right]\right\} \\
& +\left[\ln \left(-\rho_{1}+\Lambda\right)-\ln \left(-\rho_{1}-\Lambda\right)\right]\left[\ln \left(\rho_{2}+\Lambda\right)-\ln \left(\rho_{2}-\Lambda\right)\right] \\
& \times\left\{\frac{i \pi}{2}\left[\sqrt{4\left(\rho_{1}-\Lambda\right)\left(\rho_{2}+\Lambda\right)-\left(\rho-\rho_{1}-\rho_{2}\right)^{2}}-i\left(\rho-\rho_{1}-\rho_{2}\right)\right]\right. \\
& +\sqrt{4\left(\rho_{1}-\Lambda\right)\left(\rho_{2}+\Lambda\right)-\left(\rho-\rho_{1}-\rho_{2}\right)^{2}} \\
& \left.\left.\times \ln \left[\frac{\sqrt{4\left(\rho_{1}-\Lambda\right)\left(\rho_{2}+\Lambda\right)-\left(\rho-\rho_{1}-\rho_{2}\right)^{2}}-i\left(\rho-\rho_{1}-\rho_{2}\right)}{2 i \sqrt{-\left(\rho_{1}-\Lambda\right)\left(\rho_{2}+\Lambda\right)}}\right]\right\}\right\} \\
& -\Theta[-\Im(\rho)]\left\{\left[\ln \left(-\rho_{1}+\Lambda\right)-\ln \left(-\rho_{1}-\Lambda\right)\right]\left[\ln \left(-\rho_{2}+\Lambda\right)-\ln \left(-\rho_{2}-\Lambda\right)\right]\right. \\
& \times \sqrt{4\left(\rho_{1}-\Lambda\right)\left(\rho_{2}-\Lambda\right)-\left(\rho-\rho_{1}-\rho_{2}+2 \Lambda\right)^{2}} \\
& \times \ln \left[\frac{\sqrt{4\left(\rho_{1}-\Lambda\right)\left(\rho_{2}-\Lambda\right)-\left(\rho-\rho_{1}-\rho_{2}+2 \Lambda\right)^{2}}-i\left(\rho-\rho_{1}-\rho_{2}+2 \Lambda\right)}{2 \sqrt{\left(\rho_{1}-\Lambda\right)\left(\rho_{2}-\Lambda\right)}}\right] \\
& +\left[\ln \left(\rho_{1}+\Lambda\right)-\ln \left(\rho_{1}-\Lambda\right)\right]\left[\ln \left(\rho_{2}+\Lambda\right)-\ln \left(\rho_{2}-\Lambda\right)\right] \\
& \times \sqrt{4\left(\rho_{1}+\Lambda\right)\left(\rho_{2}+\Lambda\right)-\left(\rho-\rho_{1}-\rho_{2}-2 \Lambda\right)^{2}} \\
& \times \ln \left[\frac{\sqrt{4\left(\rho_{1}+\Lambda\right)\left(\rho_{2}+\Lambda\right)-\left(\rho-\rho_{1}-\rho_{2}-2 \Lambda\right)^{2}}-i\left(\rho-\rho_{1}-\rho_{2}-2 \Lambda\right)}{2 \sqrt{\left(\rho_{1}+\Lambda\right)\left(\rho_{2}+\Lambda\right)}}\right] \\
& +\left[\ln \left(\rho_{1}+\Lambda\right)-\ln \left(\rho_{1}-\Lambda\right)\right]\left[\ln \left(-\rho_{2}+\Lambda\right)-\ln \left(-\rho_{2}-\Lambda\right)\right] \\
& \times\left\{\frac{i \pi}{2}\left[\sqrt{4\left(\rho_{1}-\Lambda\right)\left(\rho_{2}+\Lambda\right)-\left(\rho-\rho_{1}-\rho_{2}\right)^{2}}-i\left(\rho-\rho_{1}-\rho_{2}\right)\right]\right. \\
& +\sqrt{4\left(\rho_{1}-\Lambda\right)\left(\rho_{2}+\Lambda\right)-\left(\rho-\rho_{1}-\rho_{2}\right)^{2}} \\
& \left.\times \ln \left[\frac{\sqrt{4\left(\rho_{1}-\Lambda\right)\left(\rho_{2}+\Lambda\right)-\left(\rho-\rho_{1}-\rho_{2}\right)^{2}}-i\left(\rho-\rho_{1}-\rho_{2}\right)}{2 i \sqrt{-\left(\rho_{1}-\Lambda\right)\left(\rho_{2}+\Lambda\right)}}\right]\right\} \\
& +\left[\ln \left(-\rho_{1}+\Lambda\right)-\ln \left(-\rho_{1}-\Lambda\right)\right]\left[\ln \left(\rho_{2}+\Lambda\right)-\ln \left(\rho_{2}-\Lambda\right)\right] \\
& \times\left\{\frac{i \pi}{2}\left[\sqrt{4\left(\rho_{1}+\Lambda\right)\left(\rho_{2}-\Lambda\right)-\left(\rho-\rho_{1}-\rho_{2}\right)^{2}}-i\left(\rho-\rho_{1}-\rho_{2}\right)\right]\right. \\
& +\sqrt{4\left(\rho_{1}+\Lambda\right)\left(\rho_{2}-\Lambda\right)-\left(\rho-\rho_{1}-\rho_{2}\right)^{2}} \\
& \left.\left.\times \ln \left[\frac{\sqrt{4\left(\rho_{1}+\Lambda\right)\left(\rho_{2}-\Lambda\right)-\left(\rho-\rho_{1}-\rho_{2}\right)^{2}}-i\left(\rho-\rho_{1}-\rho_{2}\right)}{2 i \sqrt{-\left(\rho_{1}+\Lambda\right)\left(\rho_{2}-\Lambda\right)}}\right]\right\}\right\} \\
& -\frac{i}{2}\left\{\left[\ln \left(-\rho_{1}+\Lambda\right)-\ln \left(-\rho_{1}-\Lambda\right)\right]\left[\ln \left(-\rho_{2}+\Lambda\right)-\ln \left(-\rho_{2}-\Lambda\right)\right]\right.
\end{aligned}
$$




$$
\begin{aligned}
& \times\left(\rho_{1}-\rho_{2}\right)\left[\ln \left(i \sqrt{\frac{\rho_{1}+\Lambda}{\rho_{2}+\Lambda}}\right)+\ln \left(-i \sqrt{\frac{\rho_{1}-\Lambda}{\rho_{2}-\Lambda}}\right)\right] \\
& +\left[\ln \left(\rho_{1}+\Lambda\right)-\ln \left(\rho_{1}-\Lambda\right)\right]\left[\ln \left(\rho_{2}+\Lambda\right)-\ln \left(\rho_{2}-\Lambda\right)\right] \\
& \times\left(\rho_{1}-\rho_{2}\right)\left[\ln \left(-i \sqrt{\frac{\Lambda-\rho_{1}}{\Lambda-\rho_{2}}}\right)+\ln \left(i \sqrt{\frac{\Lambda+\rho_{1}}{\Lambda+\rho_{2}}}\right)\right] \\
& +\left[\ln \left(\rho_{1}+\Lambda\right)-\ln \left(\rho_{1}-\Lambda\right)\right]\left[\ln \left(-\rho_{2}+\Lambda\right)-\ln \left(-\rho_{2}-\Lambda\right)\right] \\
& \times\left\{\left(\rho_{1}-\rho_{2}\right)\left[\ln \left(\sqrt{\frac{\Lambda+\rho_{1}}{\Lambda-\rho_{2}}}\right)+\ln \left(\sqrt{\frac{\Lambda-\rho_{1}}{\Lambda+\rho_{2}}}\right)\right]\right. \\
& +\frac{\rho_{1}-\rho_{2}}{2}\left[\ln \left(-\rho_{1}-\rho_{2}+\Lambda\right)-\ln \left(-\rho_{1}-\rho_{2}-\Lambda\right)\right. \\
& \left.-\ln \left(\rho_{1}+\rho_{2}+\Lambda\right)+\ln \left(\rho_{1}+\rho_{2}-\Lambda\right)\right]+\rho_{2}\left[\ln \left(-\rho_{1}-\rho_{2}+\Lambda\right)\right. \\
& \left.\left.-\ln \left(-\rho_{1}-\rho_{2}-\Lambda\right)\right]+\rho_{1}\left[\ln \left(\rho_{1}+\rho_{2}+\Lambda\right)-\ln \left(\rho_{1}+\rho_{2}-\Lambda\right)\right]\right\} \\
& {\left[\ln \left(-\rho_{1}+\Lambda\right)-\ln \left(-\rho_{1}-\Lambda\right)\right]\left[\ln \left(\rho_{2}+\Lambda\right)-\ln \left(\rho_{2}-\Lambda\right)\right]} \\
& \times\left\{\left(\rho_{1}-\rho_{2}\right)\left[\ln \left(\sqrt{\frac{\Lambda-\rho_{1}}{\Lambda+\rho_{2}}}\right)+\ln \left(\sqrt{\frac{\Lambda+\rho_{1}}{\Lambda-\rho_{2}}}\right)\right]\right. \\
& +\frac{\rho_{1}-\rho_{2}}{2}\left[\ln \left(\rho_{1}+\rho_{2}+\Lambda\right)-\ln \left(\rho_{1}+\rho_{2}-\Lambda\right)\right. \\
& \left.-\ln \left(-\rho_{1}-\rho_{2}+\Lambda\right)+\ln \left(-\rho_{1}-\rho_{2}-\Lambda\right)\right]+\rho_{1}\left[\ln \left(-\rho_{1}-\rho_{2}+\Lambda\right)\right. \\
& \left.\left.\left.\left\{\rho_{2}-\Lambda\right)\right]+\rho_{2}\left[\ln \left(\rho_{1}+\rho_{2}+\Lambda\right)-\ln \left(\rho_{1}+\rho_{2}-\Lambda\right)\right]\right\}\right\} \mathrm{d} \rho_{1} \mathrm{~d} \rho_{2}
\end{aligned}
$$

This defines an ultradistribution in the variables $\rho$ and $\Lambda$ for

$$
|\mathfrak{I}(\rho)|>\mathfrak{I}(\Lambda)>\left|\mathfrak{I}\left(\rho_{1}\right)\right|+\left|\mathfrak{I}\left(\rho_{2}\right)\right|
$$

Let $\mathfrak{B}$ be a vertical band contained in the complex $\lambda$-plane $\mathfrak{P}$. Integral (3.1) is an analytic function of $\lambda$ defined in the domain $\mathfrak{B}$. Moreover, it is bounded by a power of $|\rho \Lambda|$. Then, $H_{\lambda}(\rho, \Lambda)$ can be analytically continued to other parts of $\mathfrak{P}$. Thus, we define

$$
\begin{gathered}
H(\rho)=H^{(0)}\left(\rho, i 0^{+}\right) \\
H_{\lambda}\left(\rho, i 0^{+}\right)=\sum_{-m}^{\infty} H^{(n)}\left(\rho, i 0^{+}\right) \lambda^{n}
\end{gathered}
$$

As in the other cases, we define now

$$
\{F * G\}(\rho)=H(\rho)
$$

as the convolution of two Lorentz invariant tempered ultradistributions.

The Feynman propagators corresponding to a massless particle $F$ and a massive particle $G$ (mass $m$ ) are, respectively, the following ultrahyperfunctions: 


$$
\begin{gathered}
F(\rho)=-\Theta[-\mathfrak{I}(\rho)] \rho^{-1} \\
G(\rho)=-\Theta[-\mathfrak{I}(\rho)]\left(\rho+m^{2}\right)^{-1}
\end{gathered}
$$

where $\rho$ is the complex variable, such that on the real axis one has $\rho=k_{1}^{2}+k_{2}^{2}+k_{3}^{2}-k_{0}^{2}$. For them, the following equalities are satisfied

$$
\begin{gathered}
\rho^{\lambda} F(\rho)=-\Theta[-\mathfrak{I}(\rho)] \rho^{\lambda-1} \\
\rho^{\lambda} G(\rho)=-\Theta[-\mathfrak{I}(\rho)]\left(\rho+m^{2}\right)^{\lambda-1}
\end{gathered}
$$

where we have used: $\left(\rho+m^{2}\right)^{\lambda} \simeq \rho^{\lambda}$, since we have chosen $m$ to be very small. On the real axis, the previously defined propagators are given by:

$$
\begin{gathered}
f(\rho)=F(\rho+i 0)-F(\rho-i 0)=(\rho-i 0)^{-1} \\
g(\rho)=G(\rho+i 0)-G(\rho-i 0)=\left(\rho+m^{2}-i 0\right)^{-1}
\end{gathered}
$$

These are the usual expressions for Feynman propagators.

Consider first the convolution of two massless propagators. We use (3.6), since here the corresponding ultrahyperfunctions do not have singularities in the complex plane. We obtain from (3.1) a simplified expression for the convolution:

$$
\left.h_{\lambda}(\rho)=\frac{\pi}{2 \rho} \int_{-\infty}^{\infty} \int_{(}-i 0\right)^{\lambda-1}\left(\rho_{2}-i 0\right)^{\lambda-1}\left[\left(\rho-\rho_{1}-\rho_{2}\right)^{2}-4 \rho_{1} \rho_{2}\right]_{+}^{\frac{1}{2}} \mathrm{~d} \rho_{1} \mathrm{~d} \rho_{2}
$$

This expression is nothing other than the usual convolution:

$$
h_{\lambda}(\rho)=(\rho-i 0)^{\lambda-1} *(\rho-i 0)^{\lambda-1}
$$

In the same way, we obtain for massive propagators:

$$
h_{\lambda}(\rho)=\left(\rho+m^{2}-i 0\right)^{\lambda-1} *\left(\rho-m^{2}-i 0\right)^{\lambda-1}
$$

These last two expressions are the ones we will use later to evaluate the graviton's self-energy.

\section{The Lagrangian of Einstein's QFT}

The EG Lagrangian is [3] [4] [5], for curvature $\boldsymbol{R}$ and $\kappa^{2}$ the gravitation's constant,

$$
\mathcal{L}_{G}=\frac{1}{\kappa^{2}} \boldsymbol{R} \sqrt{|g|}-\frac{1}{2} \eta_{\mu v} \partial_{\alpha} h^{\mu \alpha} \partial_{\beta} h^{v \beta},
$$

where Minkowski's $\eta^{\mu v}=\operatorname{diag}(1,1,1,-1)$, while $h^{\mu v}=\sqrt{|g|} g^{\mu v}$, with $g^{\mu v}$ the metric tensor. The second term in (4.1) establishes the gauge fixing. We reach here a critical stage. At it, we will proceed to perform a crucial linear approximation. This will be immediately seen to be an approximation to the graviton field. We will write

$$
h^{\mu v}=\eta^{\mu v}+\kappa \phi^{\mu v}
$$

where $\kappa^{2}$ is the gravitation's constant and $\phi^{\mu v}$ the graviton field. Our ap- 
proximation, based on [1], reads

$$
\phi^{\mu v}=\gamma^{\mu v} \phi,
$$

with $\phi$ a scalar field and where $\gamma^{\mu \nu}$ is a constant tensor which satisfies

$$
\gamma_{\mu}^{\mu}=0
$$

This approximate casting of $\phi^{\mu v}$ considerably simplifies the handling of matters without sacrifice of rigor. We write now the Lagrangian as a sum of a non-perturbative component plus an interactive one, i.e.,

$$
\mathcal{L}_{G}=\mathcal{L}_{L}+\mathcal{L}_{I},
$$

where

$$
\mathcal{L}_{L}=-\frac{1}{4} \gamma_{\mu v} \gamma^{\mu v} \partial_{\lambda} \phi \partial^{\lambda} \phi,
$$

and, up to $2^{\text {nd }}$ order, one has [3] [4] [5]

$$
\mathcal{L}_{I}=-\frac{1}{2} \kappa \gamma^{\mu v} \phi\left[\frac{1}{2} \gamma_{\rho \lambda} \gamma^{\rho \lambda} \partial_{\mu} \phi \partial_{v} \phi+\gamma_{\mu \beta} \gamma_{\lambda v} \partial^{\lambda} \phi \partial^{\beta} \phi-\gamma_{\mu \rho} \gamma_{v}^{\rho} \partial_{\lambda} \phi \partial^{\lambda} \phi\right],
$$

having made use of the constraint (4). This constraint is required in order to satisfy gauge invariance [17] For the field $\phi$. We have then, as can also be seen to happen for the considerations made in [10],

$$
\square \phi=0 \text {, }
$$

whose solution is

$$
\phi(x)=\frac{1}{(2 \pi)^{\frac{3}{2}}} \int\left[\frac{a(\boldsymbol{k})}{\sqrt{2 k_{0}}} \mathrm{e}^{i k_{\mu} x^{\mu}}+\frac{a^{+}(\boldsymbol{k})}{\sqrt{2 k_{0}}} \mathrm{e}^{-i k_{\mu} x^{\mu}}\right] \mathrm{d}^{3} k,
$$

with $k_{0}=|\boldsymbol{k}|$. Above, $a(\boldsymbol{k})$ and $a^{+}(\boldsymbol{k})$ stand for Fourier coefficients. Up to this point, we were using Einstein's Lagrangian. Its quantization begins next.

\section{Quantization of the Theory}

As usual in QFT (see for instance Visconti' celebrated book [14]), the quantum energy-momentum tensor $T_{\rho}^{\lambda}$ is cast as

$$
T_{\rho}^{\lambda}=\frac{\partial L}{\partial \partial^{\rho} \phi^{\mu \nu}} \partial^{\lambda} \phi^{\mu v}-\delta_{\rho}^{\lambda} L,
$$

and the time-component of the four-momentum is now the quantum operator

$$
\mathcal{P}_{0}=\int T_{0}^{0} \mathrm{~d}^{3} x
$$

Using (4) we have

$$
T_{0}^{0}=-\frac{1}{4} \gamma_{\mu v} \gamma^{\mu v}\left[\partial_{0} \phi \partial^{0} \phi-\partial_{j} \phi \partial^{j} \phi\right] .
$$

Consequently,

$$
\mathcal{P}_{0}=\frac{1}{4} \gamma_{\mu v} \gamma^{\mu v} \int|\boldsymbol{k}|\left[a(\boldsymbol{k}) a^{+}(\boldsymbol{k})+a^{+}(\boldsymbol{k}) a(\boldsymbol{k})\right] \mathrm{d}^{3} k .
$$


Appeal to (2.6) leads now to

$$
\begin{gathered}
{\left[\mathcal{P}_{0}, a(\boldsymbol{k})\right]=-k_{0} a(\boldsymbol{k})} \\
{\left[\mathcal{P}_{0}, a^{+}(\boldsymbol{k})\right]=k_{0} a^{+}(\boldsymbol{k}) .}
\end{gathered}
$$

From the last relation in (5.5) one gathers that

$$
|\boldsymbol{k}| a^{+}\left(\boldsymbol{k}^{\prime}\right)=\frac{\gamma_{\mu v} \gamma^{\mu v}}{2} \int|\boldsymbol{k}|\left[a(\boldsymbol{k}), a^{+}\left(\boldsymbol{k}^{\prime}\right)\right] a^{+}(\boldsymbol{k}) \mathrm{d}^{3} k
$$

The solution of this integral equation is

$$
\left[a(\boldsymbol{k}), a^{+}\left(\boldsymbol{k}^{\prime}\right)\right]=\frac{2}{\gamma_{\mu v} \gamma^{\mu \nu}} \delta\left(\boldsymbol{k}-\boldsymbol{k}^{\prime}\right) .
$$

We use now the usual definition

$$
\Delta(x-y)=\langle 0|T[\phi(x) \phi(y)]| 0\rangle .
$$

The graviton's propagator then turns out to be

$$
\Delta(x-y)=\frac{i}{(2 \pi)^{4}} \frac{2}{\gamma_{\mu v} \gamma^{\mu \nu}} \int \frac{\mathrm{e}^{i k_{\mu}\left(x^{\mu}-y^{\mu}\right)}}{k^{2}-i 0} \mathrm{~d}^{4} k .
$$

As a consequence, we can write

$$
\mathcal{P}_{0}=\frac{\gamma_{\mu v} \gamma^{\mu v}}{4} \int|\boldsymbol{k}|\left[a(\boldsymbol{k}) a^{+}\left(\boldsymbol{k}^{\prime}\right)+a^{+}\left(\boldsymbol{k}^{\prime}\right) a(\boldsymbol{k})\right] \delta\left(\boldsymbol{k}-\boldsymbol{k}^{\prime}\right) \mathrm{d}^{3} k \mathrm{~d}^{3} k^{\prime}
$$

or

$$
\mathcal{P}_{0}=\frac{\gamma_{\mu v} \gamma^{\mu v}}{4} \int|\boldsymbol{k}|\left[2 a^{+}\left(\boldsymbol{k}^{\prime}\right) a(\boldsymbol{k})+\frac{2}{\gamma_{\rho \lambda} \gamma^{\rho \lambda}} \delta\left(\boldsymbol{k}-\boldsymbol{k}^{\prime}\right)\right] \delta\left(\boldsymbol{k}-\boldsymbol{k}^{\prime}\right) \mathrm{d}^{3} k \mathrm{~d}^{3} \boldsymbol{k}^{\prime}
$$

Thus, we obtain

$$
\mathcal{P}_{0}=\frac{\gamma_{\mu v} \gamma^{\mu v}}{2} \int|\boldsymbol{k}| a^{+}(\boldsymbol{k}) a(\boldsymbol{k}) \mathrm{d}^{3} k
$$

where we have used the fact that the product of two deltas with the same argument vanishes [6], i.e., $\delta\left(\boldsymbol{k}-\boldsymbol{k}^{\prime}\right) \delta\left(\boldsymbol{k}-\boldsymbol{k}^{\prime}\right)=0$. This illustrates the fact that using Ultrahyperfunctions is here equivalent to adopting the normal order in the definition of the time-component of the four-momentum

$$
\mathcal{P}_{0}=\frac{\gamma_{\mu v} \gamma^{\mu v}}{4} \int|\boldsymbol{k}|:\left[a(\boldsymbol{k}) a^{+}(\boldsymbol{k})+a^{+}(\boldsymbol{k}) a(\boldsymbol{k})\right]: \mathrm{d}^{3} k .
$$

Now, we must insist on the fact that the physical state should satisfy the relation (see [3] [4] [5])

$$
\gamma^{\mu v} \partial_{\mu} \phi|\psi\rangle=0
$$

The ensuing theory is similar to the QED-one obtained via the quantization approach of Gupta-Bleuler. This implies that the theory is unitary for any finite perturbative order. In this theory, just one type of graviton arises, $\phi^{12}$, while in Gupta's treatment two sorts of graviton emerge. Of course, this happens for a non-interacting theory, as pointed out by Gupta. 


\section{Graviton's Self Energy}

So as to compute the graviton's self-energy (SF) we begin with the interaction Hamiltonian $\mathcal{H}_{I}$. Remark that the Lagrangian has derivative interaction terms.

$$
\mathcal{H}_{I}=\frac{\partial \mathcal{L}_{I}}{\partial \partial^{0} \phi^{\mu \nu}} \partial^{0} \phi^{\mu \nu}-\mathcal{L}_{I} .
$$

A typical term reads

$$
\Sigma_{G \alpha_{1} \alpha_{2} \alpha_{3} \alpha_{4}}(k)=k_{\alpha_{1}} k_{\alpha_{2}}(\rho-i 0)^{-1} * k_{\alpha_{3}} k_{\alpha_{4}}(\rho-i 0)^{-1},
$$

where $\rho=k_{1}^{2}+k_{2}^{2}+k_{3}^{2}-k_{0}^{2}$.

The Fourier transform of (2) becomes

$$
\begin{aligned}
\mathcal{F} & {\left[k_{\alpha_{1}} k_{\alpha_{2}}(\rho-i 0)^{\lambda-1} * k_{\alpha_{3}} k_{\alpha_{4}}(\rho-i 0)^{\lambda-1}\right] } \\
= & -\frac{2^{4(\lambda+1)}\left[\Gamma(2+\lambda)^{2}\right]}{4 \Gamma(1-\lambda)^{2}} \eta_{\alpha_{1} \alpha_{2}} \eta_{\alpha_{3} \alpha_{4}}(x+i 0)^{-2 \lambda-4} \\
& +\frac{2^{4(\lambda+1)} \Gamma(2+\lambda) \Gamma(3+\lambda)}{2 \Gamma(1-\lambda)^{2}}\left(\eta_{\alpha_{1} \alpha_{2}} x_{\alpha_{3}} x_{\alpha_{4}}+\eta_{\alpha_{3} \alpha_{4}} x_{\alpha_{1}} x_{\alpha_{2}}\right)(x+i 0)^{-2 \lambda-5} \\
& -\frac{2^{4(\lambda+1)} \Gamma(3+\lambda)^{2}}{\Gamma(1-\lambda)} x_{\alpha_{1}} x_{\alpha_{2}} x_{\alpha_{3}} x_{\alpha_{4}}(x+i 0)^{-v-2}
\end{aligned}
$$

where $x=x_{1}^{2}+x_{2}^{2}+x_{3}^{2}-x_{0}^{2}$.

Anti-transforming the above equation one has

$$
\begin{aligned}
& k_{\alpha_{1}} k_{\alpha_{2}}(\rho-i 0)^{\lambda-1} * k_{\alpha_{3}} k_{\alpha_{4}}(\rho-i 0)^{\lambda-1} \\
& =\frac{i \pi^{2}}{4 \Gamma(1-\lambda)^{2}}\left\{\Gamma(\lambda+2)\left[\frac{\Gamma(2+\lambda)}{\Gamma(2 \lambda+4)}-2 \frac{\Gamma(3+\lambda)}{\Gamma(2 \lambda+5)}\right] \eta_{\alpha_{1} \alpha_{2}} \eta_{\alpha_{3} \alpha_{4}}\right. \\
& \left.+\frac{\Gamma(\lambda+3)^{2}}{\Gamma(2 \lambda+6)}\left(\eta_{\alpha_{1} \alpha_{2}} \eta_{\alpha_{3} \alpha_{4}}+\eta_{\alpha_{2} \alpha_{3}} \eta_{\alpha_{1} \alpha_{4}}+\eta_{\alpha_{2} \alpha_{4}} \eta_{\alpha_{1} \alpha_{3}}\right)\right\} \Gamma(-2 \lambda-2)(\rho-i 0)^{2 \lambda+2} \\
& +\frac{i \pi^{2} \Gamma(\lambda+3)}{2 \Gamma(1-\lambda)^{2}}\left\{\frac{\Gamma(2+\lambda)}{\Gamma(2 \lambda+5)} \Gamma(v+1)\left(\eta_{\alpha_{1} \alpha_{2}} k_{\alpha_{3}} k_{\alpha_{4}}+\eta_{\alpha_{3} \alpha_{4}} k_{\alpha_{1}} k_{\alpha_{2}}\right)\right. \\
& -\frac{\Gamma(\lambda+3)}{\Gamma(2 \lambda+6)}\left(\eta_{\alpha_{1} \alpha_{2}} k_{\alpha_{3}} k_{\alpha_{4}}+\eta_{\alpha_{1} \alpha_{3}} k_{\alpha_{2}} k_{\alpha_{4}}+\eta_{\alpha_{1} \alpha_{4}} k_{\alpha_{2}} k_{\alpha_{3}}+\eta_{\alpha_{3} \alpha_{4}} k_{\alpha_{1}} k_{\alpha_{2}}\right. \\
& \left.\left.+\eta_{\alpha_{2} \alpha_{3}} k_{\alpha_{1}} k_{\alpha_{4}}+\eta_{\alpha_{2} \alpha_{4}} k_{\alpha_{1}} k_{\alpha_{3}}\right)\right\} \Gamma(-2 \lambda-1)(\rho-i 0)^{2 \lambda+1} \\
& +\frac{i \pi^{2} \Gamma(\lambda+3)^{2}}{\Gamma(1-\lambda)^{2} \Gamma(2 \lambda+6)} k_{\alpha_{1}} k_{\alpha_{2}} k_{\alpha_{3}} k_{\alpha_{4}} \Gamma(-2 \lambda)(\rho-i 0)^{2 \lambda}
\end{aligned}
$$

\section{Computing the Self-Energy}

We proceed here to perform a $\lambda$-Laurent expansion, keeping from it just the $\lambda$ independent term [10]. We Laurent-expand (4) around $\lambda=0$ and encounter: 


$$
\begin{aligned}
k_{\alpha_{1}} k_{\alpha_{2}}(\rho-i 0)^{\lambda-1} * k_{\alpha_{3}} k_{\alpha_{4}}(\rho-i 0)^{\lambda-1} \\
=-i \frac{\pi^{2}}{4 \lambda}\left\{\frac{1}{5 !}\left(\eta_{\alpha_{1} \alpha_{2}} \eta_{\alpha_{3} \alpha_{4}}+\eta_{\alpha_{2} \alpha_{3}} \eta_{\alpha_{1} \alpha_{4}}+\eta_{\alpha_{2} \alpha_{4}} \eta_{\alpha_{1} \alpha_{3}}\right) \rho^{2}\right. \\
-\left[\frac{2}{4 !}\left(\eta_{\alpha_{1} \alpha_{2}} k_{\alpha_{3}} k_{\alpha_{4}}+\eta_{\alpha_{3} \alpha_{4}} k_{\alpha_{1}} k_{\alpha_{2}}\right)-\frac{1}{6 !}\left(\eta_{\alpha_{1} \alpha_{2}} k_{\alpha_{3}} k_{\alpha_{4}}\right.\right. \\
+\eta_{\alpha_{3} \alpha_{4}} k_{\alpha_{1}} k_{\alpha_{2}}+\eta_{\alpha_{1} \alpha_{3}} k_{\alpha_{2}} k_{\alpha_{4}}+\eta_{\alpha_{1} \alpha_{4}} k_{\alpha_{2}} k_{\alpha_{3}} \\
\left.\left.\left.+\eta_{\alpha_{2} \alpha_{3}} k_{\alpha_{1}} k_{\alpha_{4}}+\eta_{\alpha_{2} \alpha_{4}} k_{\alpha_{1}} k_{\alpha_{3}}\right)\right] \rho+\frac{8}{5 !} k_{\alpha_{1}} k_{\alpha_{2}} k_{\alpha_{3}} k_{\alpha_{4}}\right\} \\
-\frac{i \pi^{2}}{5 ! 2}\left(\eta_{\alpha_{1} \alpha_{2}} \eta_{\alpha_{3} \alpha_{4}}+\eta_{\alpha_{2} \alpha_{3}} \eta_{\alpha_{1} \alpha_{4}}+\eta_{\alpha_{2} \alpha_{4}} \eta_{\alpha_{1} \alpha_{3}}\right)\left[\ln (\rho-i 0)-\frac{137}{60}\right] \rho^{2} \\
+i \frac{\pi^{2}}{4 !}\left\{\left(\eta_{\alpha_{1} \alpha_{2}} k_{\alpha_{3}} k_{\alpha_{4}}+\eta_{\alpha_{3} \alpha_{4}} k_{\alpha_{1}} k_{\alpha_{2}}\right)\left[\ln (\rho-i 0)-\frac{11}{6}\right]\right. \\
-\frac{1}{24}\left(\eta_{\alpha_{1} \alpha_{2}} k_{\alpha_{3}} k_{\alpha_{4}}+\eta_{\alpha_{3} \alpha_{4}} k_{\alpha_{1}} k_{\alpha_{2}}+\eta_{\alpha_{1} \alpha_{3}} k_{\alpha_{2}} k_{\alpha_{4}}+\eta_{\alpha_{1} \alpha_{4}} k_{\alpha_{2}} k_{\alpha_{3}}\right. \\
+ \\
\left.\left.+\eta_{\alpha_{2} \alpha_{3}} k_{\alpha_{1}} k_{\alpha_{4}}+\eta_{\alpha_{2} \alpha_{4}} k_{\alpha_{1}} k_{\alpha_{3}}\right)\left[\ln (\rho-i 0)+\ln \pi+2 C-\frac{101}{30}\right]\right\} \\
\left.-i \frac{\pi^{2}}{30} k_{\alpha_{1}} k_{\alpha_{2}} k_{\alpha_{3}} k_{\alpha_{4}}\left[\ln (\rho-i 0)-\frac{47}{60}\right]+\sum_{n=1}^{\infty} a_{n} \lambda^{n}\right\} .
\end{aligned}
$$

The exact value of the convolution we are interested in, i.e., the left hand side of (5.5), is given by the independent term above, as everyone knows. Should the reader be unfamiliar with this scenario, we direct him/her to [10]. We now get

$$
\begin{aligned}
& \Sigma_{G \alpha_{1} \alpha_{2} \alpha_{3} \alpha_{4}}(k)=k_{\alpha_{1}} k_{\alpha_{2}}(\rho-i 0)^{-1} * k_{\alpha_{3}} k_{\alpha_{4}}(\rho-i 0)^{-1} \\
& =-\frac{i \pi^{2}}{5 ! 2}\left(\eta_{\alpha_{1} \alpha_{2}} \eta_{\alpha_{3} \alpha_{4}}+\eta_{\alpha_{2} \alpha_{3}} \eta_{\alpha_{1} \alpha_{4}}+\eta_{\alpha_{2} \alpha_{4}} \eta_{\alpha_{1} \alpha_{3}}\right)\left[\ln (\rho-i 0)+\ln \pi+C-\frac{46}{15}\right] \rho^{2} \\
& +i \frac{\pi^{2}}{4 !}\left\{\left(\eta_{\alpha_{1} \alpha_{2}} k_{\alpha_{3}} k_{\alpha_{4}}+\eta_{\alpha_{3} \alpha_{4}} k_{\alpha_{1}} k_{\alpha_{2}}\right)\left[\ln (\rho-i 0)+\ln \pi+C-\frac{8}{3}\right]\right. \\
& \quad-\frac{1}{24}\left(\eta_{\alpha_{1} \alpha_{2}} k_{\alpha_{3}} k_{\alpha_{4}}+\eta_{\alpha_{3} \alpha_{4}} k_{\alpha_{1}} k_{\alpha_{2}}+\eta_{\alpha_{1} \alpha_{3}} k_{\alpha_{2}} k_{\alpha_{4}}+\eta_{\alpha_{1} \alpha_{4}} k_{\alpha_{2}} k_{\alpha_{3}}\right. \\
& \left.\left.+\eta_{\alpha_{2} \alpha_{3}} k_{\alpha_{1}} k_{\alpha_{4}}+\eta_{\alpha_{2} \alpha_{4}} k_{\alpha_{1}} k_{\alpha_{3}}\right)\left[\ln (\rho-i 0)+\ln \pi+2 C-\frac{101}{15}\right]\right\} \rho \\
& \left.-i \frac{\pi^{2}}{30} k_{\alpha_{1}} k_{\alpha_{2}} k_{\alpha_{3}} k_{\alpha_{4}}\left[\ln (\rho-i 0)+\ln \pi+C-\frac{47}{30}\right]\right\} .
\end{aligned}
$$

We face here 1296 diagrams of this type.

\section{Axions Enter the Picture}

Axions are hypothetical elementary particles conjectured by the 1977 PecceiQuinn theory so as to tackle the strong CP problem in quantum chromodynamics. Should they exist and have low enough mass (within a certain range), they may be of some interest as putative components of cold dark matter [18]. We thus consider now a massive scalar field (axions) interacting with the graviton and the pertinent Lagrangian becomes 


$$
\begin{aligned}
\mathcal{L}_{G M}= & \frac{1}{\kappa^{2}} \boldsymbol{R} \sqrt{|g|}-\frac{1}{2} \eta_{\mu v} \partial_{\alpha} h^{\mu \alpha} \partial_{\beta} h^{v \beta} \\
& -\frac{1}{2}\left[h^{\mu v} \partial_{\mu} \psi \partial_{v} \psi+m^{2} \psi^{2}\right] .
\end{aligned}
$$

It is possible to recast the Lagrangian now as

$$
\mathcal{L}_{G M}=\mathcal{L}_{L}+\mathcal{L}_{I}+\mathcal{L}_{L M}+\mathcal{L}_{I M},
$$

where

$$
\mathcal{L}_{L M}=-\frac{1}{2}\left[\partial_{\mu} \psi \partial^{\mu} \psi+m^{2} \psi^{2}\right]
$$

so that $\mathcal{L}_{L M}$ is the Lagrangian for the axion-graviton action

$$
\mathcal{L}_{I M}=-\frac{1}{2} \kappa \gamma^{\mu v} \phi \partial_{\mu} \psi \partial_{\nu} \psi
$$

A new term in the interaction Hamiltonian appears

$$
\mathcal{H}_{I M}=\frac{\partial \mathcal{L}_{I M}}{\partial \partial^{0} \psi} \partial^{0} \psi-\mathcal{L}_{I M}
$$

\section{Graviton's Complete Self Energy}

Axions necessarily generate a new contribution to a graviton's self energy

$$
\Sigma_{\text {GM } \mu r v s}(k)=k_{\mu} k_{r}\left(\rho+m^{2}-i 0\right)^{-1} * k_{v} k_{s}\left(\rho+m^{2}-i 0\right)^{-1} .
$$

To evaluate it face the customary four-dimensional integral together with the Feynman-parameters denoted by the letter $x$. After a Wick rotation, we find

$$
\begin{aligned}
& k_{\mu} k_{r}\left(\rho+m^{2}-i 0\right)^{\lambda-1} * k_{v} k_{s}\left(\rho+m^{2}-i 0\right)^{\lambda-1} \\
& =i \int_{0}^{1} x^{-\lambda}(1-x)^{-\lambda} \int \frac{k_{\mu} k_{r}\left(p_{v}-k_{v}\right)\left(p_{s}-k_{s}\right)}{\left[(k-p x)^{2}+a\right]^{2-2 \lambda}} \mathrm{d}^{4} k \mathrm{~d} x,
\end{aligned}
$$

where

$$
a=p^{2} x-p^{2} x^{2}+m^{2} .
$$

Effecting a variables-change $u=k-p x$ we encounter

$$
\begin{aligned}
& k_{\mu} k_{r}\left(\rho+m^{2}-i 0\right)^{\lambda-1} * k_{v} k_{s}\left(\rho+m^{2}-i 0\right)^{\lambda-1} \\
& =i \int_{0}^{1} x^{-\lambda}(1-x)^{-\lambda} \int \frac{f(u, x, \mu, r, v, s)}{\left(u^{2}+a\right)^{2-2 \lambda}} \mathrm{d}^{4} u \mathrm{~d} x
\end{aligned}
$$

where

$$
\begin{aligned}
& f(u, x, \mu, r, v, s) \\
& =u_{\mu} u_{r} p_{v} p_{s}(1-x)^{2}+u_{\mu} u_{r} u_{v} u_{s}-u_{\mu} u_{s} p_{r} p_{v} x(1-x) \\
& \quad-u_{\mu} u_{v} p_{r} p_{s} x(1-x)-u_{r} u_{s} p_{\mu} p_{v} x(1-x) \\
& -u_{r} u_{v} p_{\mu} p_{s} x(1-x)+p_{\mu} p_{r} p_{v} p_{s} x^{2}(1-x)^{2}+u_{v} u_{s} p_{\mu} p_{r} x^{2} .
\end{aligned}
$$

After computing the associated integrals we find 


$$
\begin{aligned}
k_{\mu} k_{r}\left(\rho+m^{2}-i 0\right)^{\lambda-1} * k_{v} k_{s}\left(\rho+m^{2}-i 0\right)^{\lambda-1} & \frac{i \pi^{\frac{5}{2}} 2^{2 \lambda} m^{2+4 \lambda}}{16} \frac{\Gamma(-1-2 \lambda)}{\Gamma(1-\lambda)}\left(\eta_{\mu r} k_{v} k_{s}+\eta_{v s} k_{\mu} k_{r}\right) \\
& \times\left[\frac{F\left(1-2 \lambda,-1-2 \lambda, \frac{3}{2}-\lambda ;-\frac{\rho}{4 m^{2}}\right)}{\Gamma\left(\frac{3}{2}-\lambda\right)}+\frac{F\left(1-\lambda,-1-2 \lambda, \frac{5}{2}-\lambda ;-\frac{\rho}{4 m^{2}}\right)}{2 \Gamma\left(\frac{5}{2}-\lambda\right)}\right] \\
& +i \frac{i \pi^{\frac{5}{2}} 2^{2 \lambda-1} m^{4+4 \lambda}}{4}\left(\eta_{\mu r} \eta_{v s}+\eta_{\mu v} \eta_{r s}+\eta_{\mu s} \eta_{v r}\right) \\
& \frac{\Gamma(-2-2 \lambda)}{\Gamma(1-\lambda) \Gamma\left(\frac{3}{2}-\lambda\right)} F\left(-2-2 \lambda, 1-\lambda, \frac{3}{2}-\lambda ;-\frac{\rho}{4 m^{2}}\right) \\
- & i \frac{i \pi^{\frac{5}{2}} 2^{2 \lambda} m^{2+4 \lambda}}{64}\left(\eta_{\mu s} k_{r} k_{v}+\eta_{\mu v} k_{r} k_{s}+\eta_{r s} k_{\mu} k_{v}+\eta_{r v} k_{\mu} k_{s}\right) \\
\times & \frac{\Gamma(2-\lambda) \Gamma(-1-2 \lambda)}{\Gamma(1-\lambda)^{2} \Gamma\left(\frac{5}{2}-\lambda\right)} F\left(-1-2 \lambda, 2-\lambda, \frac{5}{2}-\lambda ;-\frac{\rho}{4 m^{2}}\right) \\
+ & i \frac{i \pi^{\frac{5}{2}} 2^{2 \lambda} m^{4 \lambda}}{32} k_{\mu} k_{r} k_{v} k_{s} \frac{\Gamma(3-\lambda) \Gamma(-2 \lambda)}{\Gamma(1-\lambda)^{2} \Gamma\left(\frac{5}{2}-\lambda\right)} F\left(-2 \lambda, 2-\lambda, \frac{5}{2}-\lambda ;-\frac{\rho}{4 m^{2}}\right) .
\end{aligned}
$$

\section{Computing the Self-Energy $(\lambda=0)$}

We appeal once again to a Laurent's expansion and have

$$
\begin{aligned}
& k_{\mu} k_{r}\left(\rho+m^{2}-i 0\right)^{\lambda-1} * k_{v} k_{s}\left(\rho+m^{2}-i 0\right)^{\lambda-1} \\
&= i \frac{\pi^{2}}{4 \lambda}\left\{m^{2}\left(\eta_{\mu r} k_{v} k_{s}+\eta_{v s} k_{\mu} k_{r}\right)\left[\frac{1}{3}+\frac{1}{5} \frac{\rho}{4 m^{2}}\right]\right. \\
&-m^{4}\left(\eta_{\mu r} \eta_{v s}+\eta_{\mu v} \eta_{r s}+\eta_{\mu s} \eta_{r v}\right) \times\left[\frac{1}{4}+\frac{1}{3} \frac{\rho}{4 m^{2}}+\frac{4}{15}\left(\frac{\rho}{4 m^{2}}\right)^{2}\right] \\
&-\frac{m^{2}}{4 m^{2}+k^{2}-i 0}\left(\eta_{\mu s} k_{r} k_{v}+\eta_{\mu v} k_{r} k_{s}+\eta_{r s} k_{\mu} k_{v}+\eta_{r v} k_{\mu} k_{s}\right) \\
&\left.\times \frac{k^{2}-m^{2}}{12}+\frac{m^{2}}{4}+\frac{k^{2}-m^{2}}{30} \frac{\rho}{4 m^{2}}-\frac{1}{6} k_{\mu} k_{r} k_{v} k_{s}\right\} \\
&+ i \frac{m^{2} \pi^{2}}{2}\left(\eta_{\mu r} k_{v} k_{s}+\eta_{v s} k_{\mu} k_{r}\right) \\
& \times\left[\frac{1}{3}\left(\ln m^{2}+\frac{1}{12}\right)+\frac{1}{5} \frac{\rho}{4 m^{2}}\left(\ln m^{2}+\frac{13}{15}\right)\right] \\
&+i \frac{m^{2} \pi^{2}}{30}\left(\eta_{\mu r} k_{v} k_{s}+\eta_{v s} k_{\mu} k_{r}\right) \frac{\rho}{4 m^{2}} \\
& \times\left[F\left(1,1, \frac{7}{2} ;-\frac{\rho}{4 m^{2}}\right)+\frac{1}{7} F\left(1,1, \frac{9}{2} ;-\frac{\rho}{4 m^{2}}\right)\right] \\
&-i \frac{\pi^{2} m^{4}}{4}\left(\eta_{\mu r} \eta_{v s}+\eta_{\mu v} \eta_{r s}+\eta_{\mu s} \eta_{v r}\right)
\end{aligned}
$$




$$
\begin{aligned}
& \times\left\{\left[\frac{1}{2}-\frac{2}{3} \frac{\rho}{4 m^{2}}-\frac{8}{15}\left(\frac{\rho}{4 m^{2}}\right)^{2}\right]\right. \\
& \left.\times\left(\ln m^{2}+1\right)-\frac{1}{2}\left[\frac{3}{2}-\frac{1}{9}\left(\frac{\rho}{4 m^{2}}\right)+\frac{52}{225}\left(\frac{\rho}{4 m^{2}}\right)^{2}\right]\right\} \\
& -i \frac{2 \pi^{2} m^{4}}{105}\left(\eta_{\mu r} \eta_{v s}+\eta_{\mu v} \eta_{r s}+\eta_{\mu s} \eta_{v r}\right)\left(\frac{\rho}{4 m^{2}}\right)^{3} F\left(1,1, \frac{9}{2} ;-\frac{\rho}{4 m^{2}}\right) \\
& -i \frac{\pi^{2} m^{2}\left(k^{2}-m^{2}\right)}{12\left(4 m^{2}+k^{2}-i 0\right)}\left(\eta_{\mu s} k_{r} k_{v}+\eta_{\mu v} k_{r} k_{s}+\eta_{r s} k_{\mu} k_{v}+\eta_{r v} k_{\mu} k_{s}\right) \\
& \times\left[\frac{1}{2}\left(\ln m^{2}+\frac{1}{3}\right)+\frac{1}{5}\left(\ln m^{2}+\frac{5}{6}\right) \frac{k^{2}}{4 m^{2}}\right] \\
& -i \frac{\pi^{2} m^{2}}{8\left(4 m^{2}+k^{2}-i 0\right)}\left(\eta_{\mu s} k_{r} k_{v}+\eta_{\mu v} k_{r} k_{s}+\eta_{r s} k_{\mu} k_{v}+\eta_{r v} k_{\mu} k_{s}\right) \\
& \times m^{2}\left[\left(\ln m^{2}+\frac{2}{3}\right)+\frac{k^{2}}{12}+\frac{k^{2}}{30} \frac{k^{2}}{4 m^{2}}\right]-\frac{i \pi^{2} m^{4}}{40\left(4 m^{2}+k^{2}-i 0\right)} \frac{k^{2}}{4 m^{2}} \\
& -i \frac{\pi^{2} m^{2}}{10}\left(\eta_{\mu s} k_{r} k_{v}+\eta_{\mu v} k_{r} k_{s}+\eta_{r s} k_{\mu} k_{v}+\eta_{r v} k_{\mu} k_{s}\right) \\
& \times \frac{k^{2}-m^{2}}{21\left(4 m^{2}+k^{2}-i 0\right)} F\left(1,1, \frac{9}{2} ;-\frac{\rho}{4 m^{2}}\right)\left(\frac{k^{2}}{4 m^{2}}\right)^{2} \\
& -i \frac{\pi^{2}}{12} k_{\mu} k_{r} k_{v} k_{s}\left[\left(\ln m^{2}+\frac{3}{4}\right)+\frac{k^{2}-4 m^{2}}{2\left(4 m^{2}+k^{2}-i 0\right)}\right] \\
& -i \frac{\pi^{2} m^{2}}{30} k_{\mu} k_{r} k_{v} k_{s} \frac{k^{2}-m^{2}}{4 m^{2}+k^{2}-i 0} \frac{k^{2}}{4 m^{2}} F\left(1,1, \frac{7}{2} ;-\frac{k^{2}}{4 m^{2}}\right)+\sum_{n=0}^{\infty} a_{n} \lambda^{n} \text {. }
\end{aligned}
$$

Once again, the exact result for our four-dimensional convolution is

$$
\begin{aligned}
& \Sigma_{G M \mu v r s}(k)=k_{\mu} k_{r}\left(\rho+m^{2}-i 0\right)^{-1} * k_{v} k_{s}\left(\rho+m^{2}-i 0\right)^{-1} \\
& =i \frac{m^{2} \pi^{2}}{2}\left(\eta_{\mu r} k_{v} k_{s}+\eta_{v s} k_{\mu} k_{r}\right) \times\left[\frac{1}{3}\left(\ln m^{2}+\frac{1}{12}\right)+\frac{1}{5} \frac{\rho}{4 m^{2}}\left(\ln m^{2}+\frac{13}{15}\right)\right] \\
& +i \frac{m^{2} \pi^{2}}{30}\left(\eta_{\mu r} k_{v} k_{s}+\eta_{v s} k_{\mu} k_{r}\right) \frac{\rho}{4 m^{2}} \\
& \times\left[F\left(1,1, \frac{7}{2} ;-\frac{\rho}{4 m^{2}}\right)+\frac{1}{7} F\left(1,1, \frac{9}{2} ;-\frac{\rho}{4 m^{2}}\right)\right] \\
& -i \frac{\pi^{2} m^{4}}{4}\left(\eta_{\mu r} \eta_{v s}+\eta_{\mu v} \eta_{r s}+\eta_{\mu s} \eta_{v r}\right) \times\left\{\left[\frac{1}{2}-\frac{2}{3} \frac{\rho}{4 m^{2}}-\frac{8}{15}\left(\frac{\rho}{4 m^{2}}\right)^{2}\right]\right. \\
& \left.\times\left(\ln m^{2}+1\right)-\frac{1}{2}\left[\frac{3}{2}-\frac{1}{9}\left(\frac{\rho}{4 m^{2}}\right)+\frac{52}{225}\left(\frac{\rho}{4 m^{2}}\right)^{2}\right]\right\} \\
& -i \frac{2 \pi^{2} m^{4}}{105}\left(\eta_{\mu r} \eta_{v s}+\eta_{\mu v} \eta_{r s}+\eta_{\mu s} \eta_{v r}\right)\left(\frac{\rho}{4 m^{2}}\right)^{3} F\left(1,1, \frac{9}{2} ;-\frac{\rho}{4 m^{2}}\right) \\
& -i \frac{\pi^{2} m^{2}\left(k^{2}-m^{2}\right)}{12\left(4 m^{2}+k^{2}-i 0\right)}\left(\eta_{\mu s} k_{r} k_{v}+\eta_{\mu v} k_{r} k_{s}+\eta_{r s} k_{\mu} k_{v}+\eta_{r v} k_{\mu} k_{s}\right)
\end{aligned}
$$




$$
\begin{aligned}
& \times\left[\frac{1}{2}\left(\ln m^{2}+\frac{1}{3}\right)+\frac{1}{5}\left(\ln m^{2}+\frac{5}{6}\right) \frac{k^{2}}{4 m^{2}}\right] \\
& -i \frac{\pi^{2} m^{2}}{8\left(4 m^{2}+k^{2}-i 0\right)}\left(\eta_{\mu s} k_{r} k_{v}+\eta_{\mu v} k_{r} k_{s}+\eta_{r s} k_{\mu} k_{v}+\eta_{r v} k_{\mu} k_{s}\right) \\
& \times m^{2}\left[\left(\ln m^{2}+\frac{2}{3}\right)+\frac{k^{2}}{12}+\frac{k^{2}}{30} \frac{k^{2}}{4 m^{2}}\right]-\frac{i \pi^{2} m^{4}}{40\left(4 m^{2}+k^{2}-i 0\right)} \frac{k^{2}}{4 m^{2}} \\
& -i \frac{\pi^{2} m^{2}}{10}\left(\eta_{\mu s} k_{r} k_{v}+\eta_{\mu v} k_{r} k_{s}+\eta_{r s} k_{\mu} k_{v}+\eta_{r v} k_{\mu} k_{s}\right) \\
& \times \frac{k^{2}-m^{2}}{21\left(4 m^{2}+k^{2}-i 0\right)} F\left(1,1, \frac{9}{2} ;-\frac{\rho}{4 m^{2}}\right)\left(\frac{k^{2}}{4 m^{2}}\right)^{2} \\
& -i \frac{\pi^{2}}{12} k_{\mu} k_{r} k_{v} k_{s}\left[\left(\ln m^{2}+\frac{3}{4}\right)+\frac{k^{2}-4 m^{2}}{2\left(4 m^{2}+k^{2}-i 0\right)}\right] \\
& -i \frac{\pi^{2} m^{2}}{30} k_{\mu} k_{r} k_{v} k_{s} \frac{k^{2}-m^{2}}{4 m^{2}+k^{2}-i 0} \frac{k^{2}}{4 m^{2}} F\left(1,1, \frac{7}{2} ;-\frac{k^{2}}{4 m^{2}}\right)
\end{aligned}
$$

Accordingly, our desired self-energy total is a combination of $\Sigma_{G \alpha_{1} \alpha_{2} \alpha_{3} \alpha_{4}}(k)$ and $\Sigma_{G M \alpha_{1} \alpha_{2} \alpha_{3} \alpha_{4}}(k)$.

\section{Axion's Self Energy}

The self-energy reads

$$
\Sigma^{\mu s}(k)=\frac{2 \gamma^{\mu v} \gamma^{r s}}{\gamma_{\rho \lambda} \gamma^{\rho \lambda}} k_{v} k_{r}\left(\rho+m^{2}-i 0\right)^{-1} *(\rho-i 0)^{-1} .
$$

In four dimensions, we have

$$
k_{v} k_{r}\left(\rho+m^{2}-i 0\right)^{-1} *(\rho-i 0)^{-1}=\int \frac{k_{v} k_{r}}{\left(k^{2}+m^{2}-i 0\right)\left[(p-k)^{2}-i 0\right]} \mathrm{d}^{4} k .
$$

Using the same Feynman parameters as above, we have

$$
\begin{aligned}
& k_{v} k_{r}\left(\rho+m^{2}-i 0\right)^{\lambda-1} *(\rho-i 0)^{\lambda-1} \\
& =i \int_{0}^{1} x^{-\lambda}(1-x)^{-\lambda} \int \frac{k_{v} k_{r}}{\left[(k-p x)^{2}+a\right]^{2-\lambda}} \mathrm{d}^{4} k \mathrm{~d} x,
\end{aligned}
$$

where

$$
a=\left(p^{2}+m^{2}\right) x-p^{2} x^{2} .
$$

We compute the integral (3) and encounter

$$
\begin{aligned}
& k_{v} k_{r}\left(\rho+m^{2}-i 0\right)^{\lambda-1} *(\rho-i 0)^{\lambda-1} \\
= & i \frac{\eta_{v r} m^{2+4 \lambda} \pi^{2}}{4} \frac{\Gamma(2+\lambda)}{\Gamma(1-\lambda)} \Gamma(-1-2 \lambda) F\left(-1-2 \lambda, 1-\lambda, 3 ;-\frac{\rho}{m^{2}}\right) \\
& +\frac{i k_{v} k_{r} m^{4 \lambda} \pi^{2}}{6} \frac{\Gamma(3+\lambda)}{\Gamma(1-\lambda)} \Gamma(-2 \lambda) F\left(-2 \lambda, 1-\lambda, 4 ;-\frac{\rho}{m^{2}}\right) .
\end{aligned}
$$




\section{Self-Energy Computation $(\lambda=0)$}

We Laurent-expand again, this time (5) around $\lambda=0$.

$$
\begin{aligned}
k_{v} & k_{r}\left(\rho+m^{2}-i 0\right)^{\lambda-1} *(\rho-i 0)^{\lambda-1} \\
= & i \pi^{2}\left\{\frac{1}{2 \lambda}\left(\frac{\eta_{v r} m^{2}}{4}-\frac{1}{3} k_{v} k_{r}\right)+\frac{\eta_{v r} m^{2}}{4}\left[\left(1+\frac{1}{3} \frac{\rho}{m^{2}}\right)\left(\ln m^{2}+\frac{1}{2}\right)\right.\right. \\
& \left.-\left(1+\frac{1}{6} \frac{\rho}{m^{2}}\right)\right]-\frac{k_{v} k_{r}}{3}\left(\ln m^{2}+\frac{3}{4}\right) \\
& \left.+\frac{1}{4}\left(\frac{\rho}{m^{2}}\right)\left[\frac{\eta_{v r} m^{2}}{12} \frac{\rho}{m^{2}}-\frac{k_{v} k_{r}}{3}\right] F\left(1,1,5 ;-\frac{\rho}{m^{2}}\right)+\sum_{n=1}^{\infty} a_{n} \lambda^{n}\right\}
\end{aligned}
$$

The $\lambda$-independent term yields the exact convolution result we need

$$
\begin{aligned}
\Sigma_{v r}(k)= & k_{v} k_{r}\left(\rho+m^{2}-i 0\right)^{-1} *(\rho-i 0)^{-1} \\
= & i \pi^{2}\left\{\frac{\eta_{v r} m^{2}}{4}\left[\left(1+\frac{1}{3} \frac{\rho}{m^{2}}\right)\left(\ln m^{2}+\frac{1}{2}\right)\right]\right. \\
& \left.-\left(1+\frac{1}{6} \frac{\rho}{m^{2}}\right)\right]-\frac{k_{v} k_{r}}{3}\left(\ln m^{2}+\frac{3}{4}\right) \\
& \left.+\frac{1}{4}\left(\frac{\rho}{m^{2}}\right)\left[\frac{\eta_{v r} m^{2}}{12} \frac{\rho}{m^{2}}-\frac{k_{v} k_{r}}{3}\right] F\left(1,1,5 ;-\frac{\rho}{m^{2}}\right)\right\}
\end{aligned}
$$

\section{Conclusions}

We have developed above an approximate quantum field theory (QFT) of Einstein's gravity (EG) that is both unitary and finite. This treatment considerably simplifies the mathematical handling so that it may constitute a valuable tool in the theorist's arsenal.

This paper addressed the same problem tackled in [19], with different mathematical techniques. Both papers dealt with gravity's quantization (GQ) via an approximate graviton's approach. The difference between that reference and the present paper resides in the fact that here we base our discourse on a general GQ formulation using Ultrahyperfunctions. In [19], instead, we constitute a special instance, based on Explicitly Lorentz Invariant Schwartz' Distributions (ELISD). The predictions of the two papers are similar, a remarkable fact given that the two types of mathematics involved are very different.

Our approximation consists of defining the graviton field as $\phi^{\mu v}=\gamma^{\mu v} \phi$ with $\gamma^{\mu \nu}$ a constant tensor and $\phi$ an scalar field.

Summing up, we have evaluated in finite fashion.

- A graviton's self-energy in the EG-field.

- The self-energy in the presence of a massive scalar field (axions, for example). Two sorts of diagram emerge: the original ones of the pure EG field plus the ones generated by the addition of a scalar field.

- An axion's self-energy. 


\section{Conflicts of Interest}

The authors declare no conflicts of interest regarding the publication of this paper.

\section{References}

[1] Plastino, A. and Rocca, M.C. (2020) Journal of Physics Communications, 4, Article ID: 035014. https://doi.org/10.1088/2399-6528/ab8178

[2] Plastino, A. and Rocca, M.C. (2020) Journal of Modern Physics, 11, 378-394. https://doi.org/10.4236/jmp.2020.113024

[3] Gupta, S.N. (1956) Proceedings of the Physical Society. Section A, 65, 161. https://doi.org/10.1088/0370-1298/65/3/301

[4] Gupta, S.N. (1956) Proceedings of the Physical Society. Section A, 65, 608-619. https://doi.org/10.1088/0370-1298/65/8/304

[5] Gupta, S.N. (1968) Physical Review, 172, 1303. https://doi.org/10.1103/PhysRev.172.1303

[6] Bollini, C.G., Escobar, T. and Rocca, M.C. (1999) International Journal of Theoretical Physics, 38, 2315-2332. https://doi.org/10.1023/A:1026623718239

[7] Bollini, C.G. and Rocca, M.C. (2004) International Journal of Theoretical Physics, 43, 1019-1051. https://doi.org/10.1023/B:IJTP.0000048599.21501.93

[8] Bollini, C.G. and Rocca, M.C. (2004) International Journal of Theoretical Physics, 43, 59-76. https://doi.org/10.1023/B:IJTP.0000028850.35090.24

[9] Bollini, C.G., Marchiano, P. and Rocca, M.C. (2007) International Journal of Theoretical Physics, 46, 3030-3059. https://doi.org/10.1007/s10773-007-9418-y

[10] Plastino, A. and Rocca, M.C. (2018) Journal of Physics Communications, 2, Article ID: 115029. https://doi.org/10.1088/2399-6528/aaf186

[11] Grothendieck, A. (1955) Produits Tensoriels Topologiques et Espaces Nucleaires (Memoirs of the American Mathematical Society, Number 16). American Mathematical Society, Providence. https://doi.org/10.1090/memo/0016

[12] Sebastiao e Silva, J. (1958) Mathematische Annalen, 136, 58-96. https://doi.org/10.1007/BF01350287

[13] Feynman, R.P. (1963) Acta Physica Polonica, 24, 697.

[14] Visconti, A. (1969) Quantum Field Theory. Pergamon Press, Oxford.

[15] Delbourgo, R. and Prasad, V.B. (1975) Journal of Physics G: Nuclear Physics, 1, 377. https://doi.org/10.1088/0305-4616/1/4/001

[16] Barci, D.G., Bollini, C.G. and Rocca, M.C. (1995) Il Nuovo Cimento, 108, 797-808. https://doi.org/10.1007/BF02731021

[17] Kleinert, H. (2016) Particles and Quantum Fields Free Web Version. https://doi.org/10.1142/9915

[18] Peccei, R.D. (2008) The Strong CP Problem and Axions. In: Kuster, M., Raffelt, G. and Beltrán, B., Eds., Axions: Theory, Cosmology, and Experimental Searches, Lecture Notes in Physics, Vol. 741, Springer, Heidelberg, 3-17. https://doi.org/10.1007/978-3-540-73518-2_1

[19] Plastino, A. and Rocca, M.C. (2020) Journal of High Energy Physics, Gravitation and Cosmology. (In Press) 